\title{
Gaming und Lernen
}

Wenn wir uns mit Gaming aus Sicht der Bibliotheken beschäftigen, dann müssen wir unbedingt auch einen Blick auf das Thema Gaming und Lernen werfen. Bibliotheken haben u. a. einen Bildungsauftrag und viele Mitarbeiter in Bibliotheken sehen darin eine wesentliche Aufgabe. Dies ist auch richtig. Gaming wird in diesem $\mathrm{Zu}$ sammenhang sehr oft als Freizeitaktivität oder als Zeitverschwendung gesehen. Dies führt manchmal dazu, dass die Träger von Bibliotheken und Teile des Kollegiums Gaming als ein Thema ansehen, welches nicht dem Bildungsauftrag einer Bibliothek entspricht. In wissenschaftlichen Bibliotheken kann man sich oft noch weniger vorstellen, warum Gaming und Lernen zusammengehören könnten.

Bibliotheken kommen aus einer Buchkultur. Und Lernen steht in unserer Gesellschaft sehr oft in einem direkten Zusammenhang mit dem Lesen von Büchern. Wie also kann Gaming gleichbedeutend mit Lernen sein? Um dies zu verstehen, müssen wir uns intensiver mit den Games und den Gamern bzw. deren Aufgaben und Lösungswegen befassen. Basierend auf den Beschreibungen von James Paul Gee in seinem Buch „What videogames have to teach us about literacy and learning“ und meinen eigenen Beobachtungen möchte ich den Prozess des Lernens in Computerspielen im Folgenden beschreiben.

Games sind komplexe Systeme. Natürlich ist nicht jedes Spiel so komplex wie z. B. EVE-Online. Aber selbst bei den einfachsten Spielen, müssen Spieler zuerst lernen. Sie müssen herausfinden, um was es genau geht. Was ist das Ziel des Spiels? Was sind meine Aufgaben? Wie bewege ich meine Spielfigur? Welche Abläufe sind hilfreich? Wer sind meine Gegner? Für was bekomme ich Punkte? Wen oder was brauche ich um meine Aufgabe zu erfüllen? Es gibt eine Vielzahl an Fragen, die den Spieler beschäftigen. Erst wenn der Spieler die notwendigen Kenntnisse erlangt hat, kann er erfolgreich spielen. Aber auch dann bleibt es ein Lernprozess. Denn er muss nun herausfinden, welche Strategie die richtige ist. Hierfür werden einzelne Schritte und Informationen strukturiert und hierarchisch geordnet. Die Komplexität der Fragestellungen mag von Spiel zu Spiel variieren. Jedoch ändert sich nichts an dem Grundmuster.

Das Besondere an Computergames ist aber, dass der Lernprozess durch Try-andFail durchgeführt wird. Das bedeutet, dass der Spieler über kontinuierliches Ausprobieren lernt. Fehler machen ist in diesem Zusammenhang nicht etwas Schlechtes. Es ist vielmehr ein elementarer Bestandteil des Gamings. Das bedeutet, Games sind komplexe und zugleich unbekannte Systeme, die ich mir als Spieler durch Try-andFail erschließen muss. Ausprobieren und aus den Erfahrungen lernen ist also Teil des Spiels. Manchmal beginnen Spiele mit Einführungs-Leveln. In diesen Leveln scheint man schon zu spielen. In Wirklichkeit trainiert einen das Spiel. Dieser Lernprozess findet in vielen Spielen kontinuierlich statt. Je komplexer und schwieriger die Aufgaben werden, desto wichtiger ist ein Design, welches es dem Spieler ermöglicht, das Level zu schaffen. Wenn der Sprung im Schwierigkeitsgrad zwischen zwei Leveln zu hoch ist, kann sehr schnell die Motivation verloren gehen.

Womit wir beim Thema Motivation wären. Spiele sind Motivationssysteme. Sie triggern bestimmte Bereiche unseres Gehirns und bringen uns dazu, immer weiter spielen zu wollen. Viele Spieler erleben irgendwann ein Gefühl des Abhebens, den sogenannten Flow. Man wird Teil des Spieles und schwebt quasi. Ich kenne diesen Effekt $u$. a. aus meiner Zeit als Musiker. Auch dort erlebten wir manchmal ein Abheben, eine Art Magie. Eli Neiburger wies auf seiner Vortragsreise durch Deutschland im Frühjahr 2013 auf einen interessanten Lerneffekt im Bereich der Leseförderung hin. Er erzählte, dass viele Kinder das Spiel „Pokemon“ spielen. Das Spiel besteht zu 
einem großen Teil aus Text. Man muss diesen Text lesen können um zu verstehen, welche Aufgaben man hat. Nun werden die Kinder in dem Spiel immer wieder mit Worten konfrontiert, die sie nicht lesen bzw. verstehen können. Um das Spiel erfolgreich weiterspielen zu können, fragen sie nun ihre Eltern oder jemanden aus ihrem Umfeld, was das jeweilige Wort bedeutet. Die Kinder lernen also über das Spiel eine Vielzahl neuer Worte kennen. Der Lernprozess ist Teil des Spiels und das Ergebnis ist, dass es den Kindern Spaß macht zu lernen. Sie merken letztlich noch nicht einmal, dass sie gerade lesen lernen. Aber das ist noch längst nicht alles.

Auch wenn man über Try-and-Fail ein Spiel verstehen kann, gibt es doch immer wieder Situationen, bei denen man nicht weiterkommt. Alle Versuche, das Problem zu lösen, schlagen fehl. Man benötigt weitere Informationen bzw. Sekundärinformationen, und diese findet man sehr oft im Internet. Genauer gesagt, hat die GamingCommunity ein eigenes Informationsmanagement entwickelt. $\mathrm{Zu}$ nahezu allen halbwegs erfolgreichen Spielen gibt es Foren, Wikis, Video-Tutorials etc. Gerade zu komplexen Spielen mit verschiedenen Figuren, komplexen Wirtschaftssystemen, und Aufgaben, die man in Gruppen lösen muss, gibt es Wikis. Dabei ist es egal, welche Altersgruppe das Spiel spielt. So gibt es Wikis für World of Warcraft und ebenso Wikis für Pokemon. Auch in Foren und Communities oder auf Facebook und Google+ gibt es solche Informationsplattformen. Ein besonderes Highlight sind sicherlich die Let's Play Videos, welche wir auf Youtube finden können. Hier werden Computergames live gezeigt. Manche der Spiele dauern viele Stunden, und wenn man will, kann man die Spiele von Beginn an verfolgen. Let's Play hat sich zu einer richtigen Bewegung entwickelt, und ich selber nutze dieses Angebot immer wieder.

Das spannende sind aber nicht alleine die Wikis, Foren, Videos etc. Besonders interessant ist die Tatsache, dass diese Informationen nicht durch die Unternehmen erstellt und angeboten werden, sondern durch die Gamer selber. Das Entwickeln von neuem Know-How und das Teilen dieser Informationen sind ein elementarer Bestandteil der Gaming-Kultur. Das mag auf den ersten Blick wenig nachvollziehbar sein. Warum behalten die Gamer ihr Wissen nicht für sich und haben so einen Vorteil gegenüber den anderen? Der Grund dafür ist der, dass Gamer in den meisten Fällen einen Wettbewerb auf Augenhöhe erleben wollen. Gerade wenn man Spiele im Multiplayer-Modus spielt, was bedeutet, dass man gegen reale Gamer antritt und nicht gegen den Computer spielt, wird versucht, dass immer gleich starke Spieler gegeneinander spielen können. Ich selber habe schon erlebt, dass ich aus Spielen „entfernt“ wurde, weil ich einen zu niedrigen Level hatte. Es geht also nicht alleinig darum zu siegen, sondern man möchte gegen gleich starke Sieger spielen. Des Weiteren ist der von mir schon benannte soziale Charakter bzw. die Gaming-Community bedeutsam. Auch wenn man gegeneinander spielt, ist man doch als Gamer mit den Anderen vereint.

Bis jetzt haben wir uns mit den Gamern, den Games und den Begriffen Lernen und Information beschäftigt. Ein anderer Punkt betrifft nicht die Spiele an sich, sondern die damit verbundenen Denk-, Lern- und Arbeitsweisen. Wie bereits im Beispiel von Pokemon beschrieben, spielt Motivation eine wesentliche Rolle beim Gaming und damit verbunden beim Lernen. Spielen ist Arbeit. Ich muss lernen und trainieren, ich muss mir Sekundärinformationen besorgen und wenn möglich auch zur Verfügung stellen. Und je nachdem, wie komplex das Spiel ist, ist dieses Lernen und Arbeiten sehr zeitaufwendig. In manchen Spielen sind die Informationsgewinnung und die Analyse der aktuellen Situation aufwendiger und zeitintensiver als das Spielen selbst. Warum tun Gamer so etwas? Warum beschäftigen sie sich so lange damit? Warum ist es plötzlich für Kinder kein Problem, 700 verschiedene Pokemons und deren Funktionen und Nutzungsoptionen zu erlernen? Warum wird das Durcharbeiten von Excel-Listen, Wikis und Tutorials plötzlich interessant? Die Antwort ist einfach: Weil es Spiele sind. Spiele 
funktionieren nach einem eigenen System. Sie triggern bestimmte Bereiche unserer Psyche. Es macht Spaß zu lernen und zu arbeiten. Genau diesen Effekt versuchen viele Unternehmen und Institutionen sich zu Nutze zu machen. In Lern- und Arbeitsprozessen werden Elemente bzw. Modelle aus dem Bereich Gaming implementiert. Zudem werden Spiele entworfen, die das Ziel haben, bestimmte Inhalte zu lehren.

\section{Zusammenfassung}

Gaming hat immer etwas mit Lernen zu tun. Gamer werden kontinuierlich darauf trainiert, ihnen unbekannte und in den meisten Fällen komplexe Systeme zu erschließen und darin bestimmte Aufgaben zu erfüllen. Sie müssen selber herausfinden, wie das System funktioniert, welche Aufgaben Sie haben, wie Sie diese erfüllen können, welche Ressourcen inkl. Mitspieler dafür benötigt werden, welche Lösungsstrategien funktionieren und welche Konsequenzen diese Entscheidungen auf alle Bereiche des Systems haben. Sie müssen zudem moderne Kommunikations- und Medientechnologien beherrschen, um Sekundärinformationen zu bekommen bzw. zu erstellen und zu teilen. Dies alles funktioniert in einem kontinuierlich fortschreitenden Prozess. Aber ist das nicht zumindest in Teilen genau das, was Kinder und Jugendliche in der Schule lernen sollen? Und erwarten wir dies nicht auch von Mitarbeitern in Unternehmen?

Alle diese Aktivitäten werden von den Gamern freiwillig umgesetzt, ja sie haben sogar Spaß dabei. In vielen Punkten lernen sie auch schreiben und lesen, denn die meisten Spiele haben einen größeren Anteil an Text - entweder im Spiel oder bei der Recherche nach Sekundärinformationen - und dieser Text muss komplett verstanden werden. Die Motivationsmodelle und der spielerische Umgang mit den jeweiligen Inhalten, sowie die Vernetzung mit modernen Kommunikations- und Medientechnologien sorgen dafür, dass immer öfter versucht wird, die Systeme und Modelle auf andere Bereiche zu übertragen. Sei es, in dem man spezielle Spiele zum Lernen von bestimmten Inhalten entwickelt, oder sei es, dass man nur die Mechaniken und Denkweisen in neue Arbeitsprozesse implementiert. Schließlich gibt es auch noch Beispiele, bei denen beispielsweise mit normalen Spielen versucht wird, das Verstehen komplexer Systeme, die Vernetzung von Informationen, Sprachen etc. zu lehren. Wenn Sie diese genannten Punkte akzeptieren können, dann wird hoffentlich klar, dass Gaming mehr ist als nur ein netter Zeitvertreib und dass sich Bibliotheken u. a. gerade aus dem Blickwinkel des Bildungsauftrags mit dem Thema befassen sollten. Gaming ist Lernen. Und Bibliotheken können mit Gaming neue Lernformen unterstützen. Natürlich ist Gaming nicht der Heilsbringer für alles. Gaming kann nicht das Lesen von Büchern ersetzen und natürlich gibt es Inhalte und Fakten, die wir lernen müssen. Zudem gibt es Menschen, die einfach nicht für Gaming geschaffen sind. Es geht also nicht um ein „entweder oder“ sondern um ein „sowohl als auch“. Gerade in einer Welt, in der es nahezu unendlich viele Informationen gibt, und in der von immer mehr Menschen erwartet wird, zu einem selbstlemenden System zu werden, welches sich und andere motiviert und unterstützt, ist Gaming ein möglicher Schlüssel für einen nachhaltigen Lernerfolg.

\section{Gaming als Kulturgut}

In den letzten Jahren wurde sehr viel über das Thema Gaming diskutiert. Eine Fragestellung war die, ob Gaming ein Kulturgut sei oder nicht. Diese Frage mag auf den ersten Blick als nicht besonders relevant erscheinen. Gaming ist ein Element unserer Alltagskultur, so wie Filme oder Pop-Musik. Trotzdem gab es auch hier teils hitzige 
Diskussionen. Die Befürchtungen waren vielfältig. Einige störten sich an der Tatsache, dass die Gaming-Welt mehr oder weniger komplett kommerziell ist. Andere fragten sich, ob es nicht eine Abgrenzung zu Theatern und Museen geben müsste. Wiederum andere meinten, dass Gaming schlecht für die Menschen sei und man so etwas nicht auch noch legitimieren müsse. Gerade in dem zuletzt genannten Argument spiegelt sich eine bis heute in vielen Kulturbereichen vorhandene Ablehnung bezüglich der digitalen Welt bzw. allem Neuen wieder. Auch die Kulturpolitik ist in Teilen noch immer eine konservative Welt. Andererseits wurde und wird seit Jahren versucht, Gaming zum Beispiel im Bereich der kulturellen Bildung als relevant zu definieren. Letztlich waren es ein paar Entscheidungen welche u.a. durch den Deutschen Kulturrat und seinen Geschäftsführer Olaf Zimmermann angeschoben wurden, die dafür sorgten, dass Gaming auch im Kultursektor endlich angenommen wurde. Mit dem Deutschen Computerspielepreis, welcher seit 2011 durch die Branchenverbände BIU und G.A.M.E. sowie dem Beauftragten der Bundesregierung für Kultur und Medien, Staatsminister Bernd Neumann ausgerichtet wird, hat das Thema zumindest nach außen seinen Platz in der Kultur gefunden. Das sollte aber nicht darüber hinwegtäuschen, dass es auch aus dem Kultursektor noch immer große Kritik an diesem Thema und auch am Preis gibt. Viele Kritiker stört es, dass der Preis keinen pädagogischen Ansatz verfolgt und damit Spiele wie Crysis 2 (ein Ego-Shooter) ebenfalls ausgezeichnet wurden. Selbst die Tatsache, dass es ohne den Bereich Gaming wohl kaum eine so große Bedeutung der Kultur- und Kreativwirtschaft geben würde, hat bis jetzt nichts daran geändert. Beide Ebenen, die Ablehnung der digitalen Kultur im Allgemeinen und der Gaming-Kultur im Speziellen sind nach wie vor im Kultursektor vorhanden und werden wohl auch weiterhin bestehen. Dabei könnte der Kultursektor von der Welt des Gamings einiges lernen.

Unabhängig von der kulturpolitischen Diskussion sind m.E. Computergames noch aus einem anderen Grund als Kulturgut zu bezeichnen. Sie sind Kunstwerke. Bezüglich Ästhetik stehen viele Games anderen Kulturformen in nichts nach. Ihre Darstellungen von Phantasiewelten sind beeindruckend. Sie sind ebenso Bühnen für große Geschichten. Ihre Figuren und Geschichten sind letztlich unserer Kultur bzw. regionalen Kulturen entnommen. So wirkt manches Spiel wie z. B. „World of Warcraft“ oder „the Elder Scrolls“ wie eine moderne Wagner-Oper. Spiele wie „Deus X - Human Revolution“ scheinen wie eine Weiterentwicklung von Geschichten wie „Blade Runner“. Und „Mafia 2“ wirkt wie „Der Pate“ oder „The Godfellas“. Das Besondere an dieser Kunstform ist aber, dass der Rezipient Teil des Werkes wird. Wir konsumieren nicht nur, sondern wir erleben und sind aktiv Teil der Geschichte. Dies hat auch einen Einfluss auf die Kunstform bzw. die Arbeit des Künstlers. Viele Kunstformen entstehen letztlich ohne eine Fokussierung auf den Rezipienten. Kunst soll und darf für sich alleine stehen. Sie muss nicht wahrgenommen werden bzw. demjenigen, der sie wahrnimmt, nicht gefallen. Gaming ist hier anders. Zwar kann man auch hier verstören oder gar polarisieren, jedoch ist immer ein Gameplay erforderlich, welches den Rezipienten motiviert, das Spiel zu spielen. Ein Spiel, welches nicht gespielt wird, existiert nicht. Die Vermittlung des kulturellen Inhalts ist also Teil des Werkes und nicht wie sonst davon getrennt.

Aber nicht nur die Spiele selbst sind Kunstwerke. Auch um die Spiele herum entsteht so einiges. So gibt es die Artwork-Ausstellung „Pic your game life“ des Instituts Spawnpoint. In den großflächigen Gemälden zeichnen sich Gamer selbst als Figur in ihrem Lieblingsspiel. Die Musik aus Computergames ist ebenfalls kulturell bedeutend. Die in Leipzig stattgefundene Gamesconvention, welche der Vorläufer der Gamescom in Köln war, wurde mit einem Konzert im Leipziger Gewandhaus eröffnet, bei dem die besten Musikstücke aus den Spielen des vergangenen Jahres aufgeführt 
wurden. Die Geschichten, welche in Spielen erzählt werden, bilden immer öfter die Basis für Filme und Bücher. Und natürlich gibt es auch Games, die die Geschichten aus Filmen und Büchern weiter erzählen.

\section{Zusammenfassung}

Gaming ist ein Kulturgut. Die Anerkennung als Kulturgut wird aber noch immer kontrovers diskutiert. Computergames sind sehr oft selbst Kunstwerke, die aber ein entscheidendes Alleinstellungsmerkmal haben: In den meisten Fällen soll Kunst keine Funktion haben. Das Werk muss nicht gefallen, es muss sich nicht verkaufen, es ist Ausdruck des Künstlers und es steht für sich. Computerspiele haben jedoch einen direkten Bezug zum Rezipienten. Computerspiele funktionieren erst dann, wenn sie gespielt werden. Das bedeutet, dass der „Künstler“ das Spielen, also die aktive Handlung inkl. Motivationsmodellen, Storytelling etc., in das Spiel mit einbauen muss. Es gibt eine Vielzahl an sogenannten Indie-Games, die nichts weiter als kleine Kunstwerke sind.

Computerspiele sind aber ebenso Spiegel der kulturellen Identität einer Gesellschaft. In der Visualisierung von Inhalten, in den erzählten Geschichten, in der Nutzung der Spiele können wir die einzelnen kulturellen Strömungen ganzer Gesellschaften wieder erkennen. Einzelne Elemente der Spiele beeinflussen auch andere Kunstformen oder werden von anderen Kunstformen beeinflusst. Die Musik aus manchen Computerspielen wird von internationalen Orchestern aufgeführt. Es gibt Bücher und Filme, welche auf Filmen basieren und umgekehrt. Es gibt Ausstellungen von Malern, die einzelne Räume aus Spielen auf Leinwand verewigt haben, und selbst die großen Museen und Bibliotheken dieser Welt beginnen mit dem Aufbau von eigenen Sammlungen. Wenn Bibliotheken Lern- und Kulturorte sind, dann gehören Games definitiv mit dazu.

\section{Gaming als Element der digitalen Gesellschaft}

Gaming und Lernen bzw. Gaming als Kulturgut, dies alles mag für Sie nachvollziehbar erscheinen. Aber was hat Gaming mit der digitalen Gesellschaft zu tun? In den letzten 20 Jahren hat sich unsere Medien- und Kommunikationswelt nachhaltig verändert. Das Internet, Social Media oder das mobile Internet stehen für einen tiefgreifenden Wandel. Es geht längst nicht nur um neue Technologien. Es geht vielmehr um das Entstehen einer neuen Kultur, die zunehmend alle Gesellschaftsbereiche verändert. In der Wahrnehmung dieses Wandels werden die Computergames gerne übersehen. Sie gelten noch immer als ein Phänomen vorwiegend männlicher Jugendlicher. Sie erscheinen wie eine Nische. Und diese Wahrnehmung wird vor allem in den Communities verstärkt, deren Fokus auf anderen Medien liegt. Allenfalls wird Gaming als Wirtschaftsfaktor angesehen, aber auch das passiert eher in kleinen Communities wie z. B. der Kultur- und Kreativwirtschaft.

Warum Gaming in der Diskussion nur eine untergeordnete Rolle spielt, lässt sich schwer sagen. Ich behaupte jedoch, dass es ohne Gaming überhaupt keine digitale Gesellschaft geben würde bzw. geben kann. Es sind Computerspiele, die vielen Menschen einen ersten Kontakt zu Computern etc. ermöglichen. Es sind Computerspiele, die eine kontinuierliche Nutzung von Smartphones und Tablet-PCs befördern. Es sind nicht die vielen Arbeitswerkzeuge und auch nicht die vielen Informationsplattformen. Gaming ist in diesem Zusammenhang nichts anderes als der spieleri- 
sche Umgang mit Hochtechnologie. Die besten Communitymanager finden wir in der Games-Industrie. Neue Technologien wie Augmented Reality sind schon seit langem Bestandteil verschiedener Games. Games befördern zudem den Ausbau der digitalen Infrastruktur. Plattformen wie Facebook haben lange Zeit einen erheblichen Teil ihrer Umsätze durch Spiele generiert. Die Weiterentwicklung des Heimcomputers ist maßgeblich durch Gaming angetrieben worden.

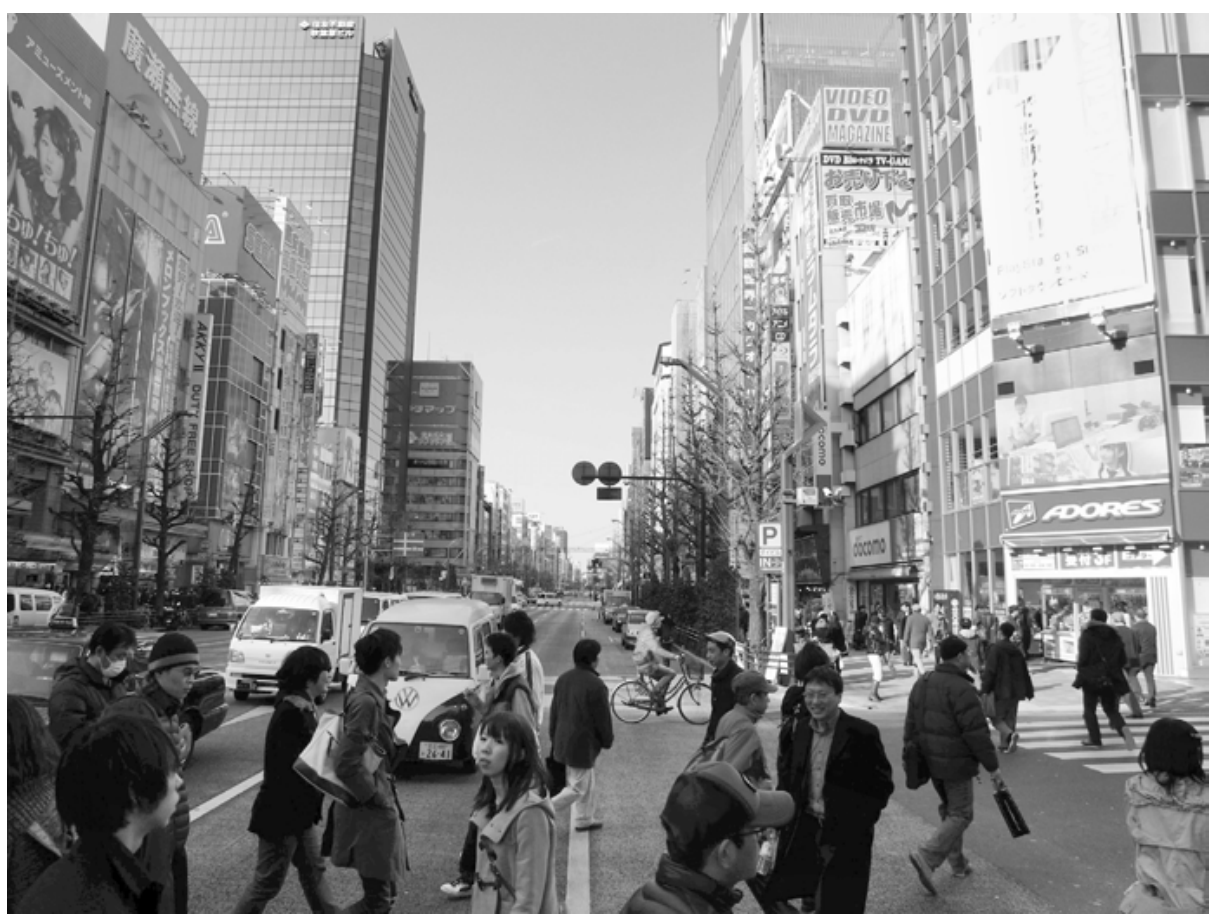

Abb. 4 Blick über die Elektronikmeile „Akihabara“ in Tokio.

Aber Gaming ist nicht nur ein Antriebsmotor für die spielerische Digitalisierung einer Gesellschaft. Gaming ist zudem ein Indikator für den Grad der Digitalisierung einer Gesellschaft. Dies betrifft zum einen die digitale Infrastruktur und zum anderen die Implementierung der digitalen Kultur. Die Akzeptanz der digitalen Kultur ist ein wesentliches Kriterium für den Zustand einer digitalen Gesellschaft. Nimmt die Bevölkerung die neuen Angebote und Plattformen an? Es reicht bei weitem nicht aus, nur eine digitale Infrastruktur aufzubauen. Es ist ebenso wichtig, der Bevölkerung diese neuen Möglichkeiten näher zu bringen.

Wenn sich Bibliotheken dem Thema Gaming widmen, dann öffnen sie sich auch einer neuen Kultur und neuen Technologien. Sie schaffen nicht nur den Zugang zu Inhalten oder Medien sondern auch zu neuen Denk- und Arbeitsweisen. Gaming in Bibliotheken ist also mehr als nur die Beschäftigung mit etwas Neuem. Es bedeutet, dass Gaming eine neue Relevanz bekommt. Die Bibliothek als Ort ist m.E. im 21. Jahrhundert ohne Gaming überhaupt nicht denkbar.

Schließlich wird Gaming auch nach innen wirken. Es entstehen intern wie extern neue Communities und früher oder später ein neues Selbstverständnis der Bibliothek. Wenn Sie Gaming ernst nehmen, dann nehmen Sie auch den digitalen Teil unserer Gesellschaft wahr. 


\section{Gaming und alles darum}

Sie haben nun einen ersten Einblick in das Thema Gaming bekommen, und ich hoffe, Sie verstehen die Gaming-Kultur und ihre Relevanz jetzt ein bisschen besser. Und trotzdem gibt es noch viel mehr in der Welt des Gamings zu entdecken. Besonders spannend ist auch das Gaming-Umfeld. Gamer sitzen nicht einfach vor ihrem Computer oder ihrer Konsole und spielen. Gaming war und ist noch immer eine sehr soziale Aktivität. Die Vernetzung unter den Gamern findet nicht nur in der digitalen Welt statt. Viele Gamer treffen sich auch in der ,analogen Welt“, um sich auszutauschen oder gemeinsam zu spielen. In manchen Spielen sind Gamer in Gilden oder Clans organisiert. Sie lösen gemeinsam Aufgaben oder treten gemeinsam in Wettbewerben an. Sie treffen sich in der realen Welt. Sie besuchen Ausstellungen mit Gemälden aus verschiedenen Spielen. Oder sie treffen sich auf LAN-Partys, bei denen sie ihre Computer mitbringen, um zusammen zu spielen. In anderen Fällen treffen sie sich, um gemeinsam mobile Games zu spielen.

Ein besonderes Highlight ist für mich die Gamescom, zu der einige Spieler sogar in Kostümen ihrer Spielfiguren kommen. Diese Kostüme sind sehr oft äußerst hochwertig und von den Spielern selbst hergestellt worden. Es gibt Spieler die bis zu $10.000 €$ und Hunderte an Arbeitsstunden in ihr Kostüm investieren.

Nun werden Sie vielleicht einwenden, dass dieses Verhalten übertrieben sei. In vielen meiner Workshops ernte ich vor allem Kopfschütteln, wenn es um diese Verbindung zu Games bzw. den damit verbundenen Figuren geht. Aber wenn man genau hinsieht, stellt man fest, dass es solche Phänomene auch in anderen Kulturbereichen gibt. Die Zeit und die finanziellen Mittel, die manche Menschen in den Karneval stecken, sind ebenfalls beeindruckend. Und wie viele Menschen campierten vor mancher Buchhandlung, nur um so früh wie möglich den neuen Harry Potter-Roman zu kaufen. Selbst in der sogenannten Hochkultur - der Begriff ist an sich dämlich und schadet unserer Kultur kontinuierlich - gibt es eigene Rituale und Kleiderordnungen. Die Gaming-Kultur ist nicht exotischer oder besser oder schlechter als jede andere Kulturform. Sie ist aber eine große Chance für Bibliotheken.

Wenn sich nun Bibliotheken mit dem Thema Gaming befassen, dann sollten sie zuerst nicht auf die Spiele oder die eine oder andere Zielgruppe schauen. Es ist viel wichtiger, dass Sie die Gaming-Kultur als Ganzes verstehen und überlegen, wo Sie als Bibliothek einen Raum für diese Kultur schaffen können. Viele der Herausforderungen, welche Sie im Laufe der Beschäftigung mit dem Thema bewältigen müssen, erledigen sich leichter, wenn Sie bereits verstanden haben, was Gaming bedeutet, und Sie zudem eine Plattform für Gamer sein wollen. Es ist wichtig, dass Sie Gaming nicht als Mittel zum Zweck (z. B. um Menschen zum Lesen von Büchern zu animieren) benutzen. Akzeptieren Sie das Thema als gleichwertig zu Büchern und zu allen Ihren anderen Aktivitäten.

Beschäftigen Sie sich mit der Gaming-Kultur. Sie müssen kein Hardcore-Gamer werden. Aber das Wissen und das Verständnis über das Thema Gaming gibt Ihnen auch die Möglichkeit, argumentativ auf eventuelle Kritik zu reagieren. Wenn Sie z. B. von Ihrem Träger oder von Eltern kritisiert werden, ist es wenig hilfreich, wenn Sie in den Chor mit einstimmen. Kurz gesagt: So wie eine Bibliothek ein Botschafter der Bücher bzw. des Lesens ist, sollte sie es auch bezüglich Games und Gaming sein. 


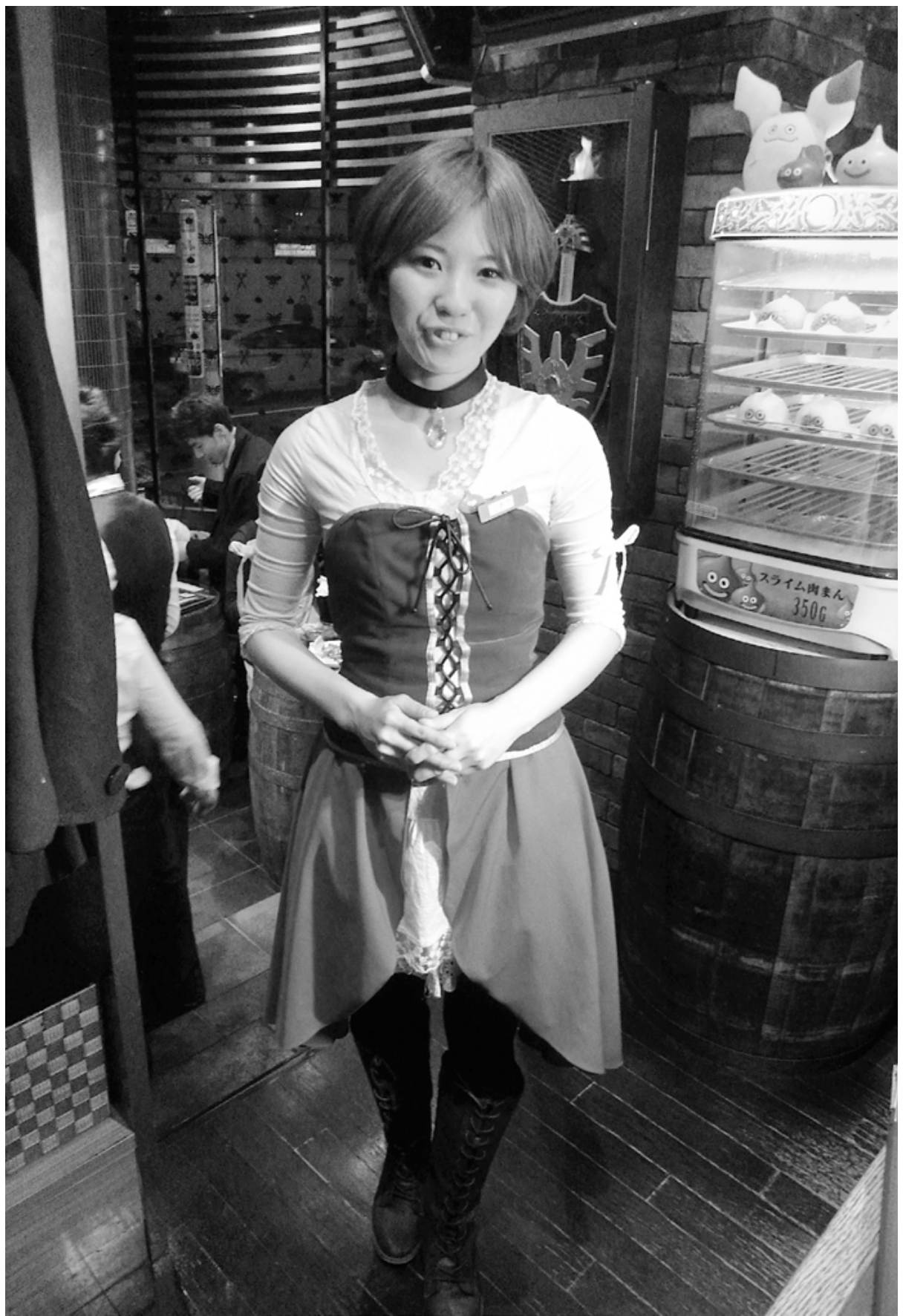

Abb. 5 Servicekraft im Restaurant „Luidas Bar“ in Tokio. Das Restaurantkonzept basiert hinsichtlich Ausstattung, Service, Essen und Getränke auf dem Spiel Dragon Quest. 